\title{
Dual-cap Mushroom-like Metasurface used in CP Reconfigurable Monopole Antenna for Performance Enhancement
}

\author{
Y.F. Cao, Student Member, IEEE, S.W. Cheung, Senior Member, IEEE, and T.I. Yuk, Member, IEEE
}

\begin{abstract}
A dual-cap mushroom-like metasurface (MS) is proposed. The proposed MS is called dual-cap mushroom-like MS because each unit cell has a simple patch with a centered via on one side of the substrate and a meandered slot on the other side, while conventional mushroom-like MS has one side of the substrate being a plain conducting plate. The operating frequency and bandwidth of the unit cells are analyzed using an equivalent circuit which is verified using the software tools Advanced Design System (ADS) and CST. Results show that the meander slot on the other side of the substrate introduces inductive effects to lower down the operating frequency and increase the operating bandwidth of the unit cells. Comparison is made with other unit cells. The proposed MS is then used as a reflector in the design of a planar circular-polarization reconfiguration (CPR) monopole antenna for the Global Navigation Satellite System (GNSS). Simulation results show that, by placing at only $0.09 \lambda_{0}$ from the antenna, the MS can increase the boresight gain and the operating bandwidth from 0.4 to $6.6 \mathrm{dBi}$, and 1.46-1.79 $\mathrm{GHz}$ to $1.42-1.88 \mathrm{GHz}$, respectively.
\end{abstract}

Index Terms-Metasurface, circular polarization, reconfigurable, monopole antenna, biasing circuit.

\section{INTRODUCTION}

$\mathrm{M}$ ETASURFACE (MS) is a two-dimensional equivalent metamaterial and can be implemented using small unit cells [1]. With the succinct planar structure, MS can be used in the design of planar antennas with improved performances. example, in [2, 3], a MS was placed in front of a patch/slot/monopole antenna to improve the boresight gain or convert linear polarization (LP) to circular polarization (CP). [4-12], a MS was placed at the back of a patch/monopole antenna to enhance the boresight gain. At low operating frequencies such as $1.58 \mathrm{GHz}$ for the Global Navigation Satellite System (GNSS), the required size of the unit cell in [4-8] would be very large. To reduce the required size, unit with different shapes such as uniplanar compact photonic bandgap (UC-PBG) lattice [9], waffle-like [10], square loop [11] and Minkowski fractal mushroom-like [12] were proposed. All these unit cells [4-12] had the same problem of narrow operating bandwidths. Designing a small unit cell for

Y.F. Cao, S. W. Cheung, and T. I. Yuk are with the Department of Electrical and Electronic Engineering, The University of Hong Kong, Pokfulam Road, Hong Kong, China (email: [yfcao, swcheung, tiyuk]@eee.hku.hk). low frequency operation is one of the objectives of our studies here.

$\mathrm{CP}$ reconfigurable (CPR) antenna can be switched between right- and left-handed CP (RHCP and LHCP) and finds many applications such as synthetic-aperture-radar, multiple-inputmultiple-output (MIMO) systems and frequency reuses [13]. Usually, CPR antennas are designed using patch antennas or slot antennas with PIN diodes as switches [14-27]. For CPR patch antennas, the reconfigurability between RHCP and LHCP is normally achieved using four methods, reconfiguring the radiating-patch shapes using PIN-diode switches [14-16], etching slots with PIN-diode switches on the patch radiators $[17,18]$, placing PIN-diode switches on ground-plane slots $[19,20]$, and designing reconfigurable microstrip feeding networks using PIN-diode switches [21, 22]. When using PIN diodes as switches in CPR antennas, the DC basing circuits have to be carefully designed in order not to affect the antenna performances. Thus in [16], the PIN diodes were biased using a bias- $T$ to avoid the effects of the DC circuit on the antenna RF performance. In $[19,20]$, the DC circuits were all placed on the ground planes to reduce the effects on the antenna performances. For CPR slot antennas, the CP reconfigurability is usually achieved using three methods, i.e., changing the shapes of the radiating slots using PIN-diode switches [23, 24], reconfiguring the feeding networks of the radiating slot using PIN-diode switches $[25,26]$, and switching ON/OFF the right/left $T$-shaped microstrips protruding from the ground plane using PIN-diode switches [27]. The biasing circuits for the slot antennas in [23-27] were placed on the ground plane and so had little effects on the antenna performances. Recently, it was also proposed to use MS with a slot antenna to design a CP antenna [28].

Compared with patch and slot antennas which require an electrical length of $\lambda_{g} / 2$, monopole antenna requires a shorter electrical length of $\lambda_{g} / 4$ (where $\lambda_{g}$ is the guided wavelength at the center frequency) and has wider bandwidth. Thus a CPR monopole antenna was proposed in [29] which had a smaller size and wider axial-ratio bandwidth (ARBW, AR $<3 \mathrm{~dB}$ ) than all the designs in [14-28]. However, it had a very low realized boresight gain of about $0 \mathrm{dBi}$ and a complicated switchable feeding network. Simplification of this CPR antenna is another objective of our studies.

In this paper, a dual-cap mushroom-like MS is proposed 
used in the design of a compact CPR monopole antenna for the GNSS system. Conventional mushroom-like MS has one side of the substrate being a plain conducting plate. However, each unit cell on the proposed MS has a simple patch with a centered via on one side of the substrate and a meandered slot on the other side, so it is here called a dual-cap mushroom-like unit cell. The unit cell is analyzed using an equivalent circuit which is verified using the circuit simulation tool Advanced Design Systems (ADS) and EM simulation tool CST. The operating frequency and bandwidth of the dual-cap mushroom-like unit cell are compared with other unit cells proposed in [4-12]. Results show that the meander slot on the other side of the unit cell introduces inductive effects which can lower down the operating frequency and increase the operating bandwidth, thus the unit cell requires a smaller required size. The dual-cap mushroom-like MS is used in the design of a CPR planar antenna with enhanced boresight gain and bandwidth. The MS is placed at a short distance of only $0.09 \lambda_{0}$ away from a CPR antenna and serves as a reflector. In such setup, the CPR antenna is called the source antenna and the CPR antenna together with the MS is called a CPR MS antenna $[2,3]$. The CPR antenna is similar to our previous design in [29], but with much reduced complexity. The CPR MS antenna is studied and designed using the EM simulation tool CST. For validation of simulation, the CPR MS antenna is fabricated and measured using the Satimo Starlab system [30]. Simulation results show that the dual-cap mushroom-like MS can increase the operating bandwidths of the CPR antenna from $1.46-1.79 \mathrm{GHz}(0.33 \mathrm{GHz}, 20.3 \%)$ to 1.42 to $1.88 \mathrm{GHz}$ $(0.46 \mathrm{GHz}, 27.9 \%)$ for the GNSS system, and also the boresight gain at $1.58 \mathrm{GHz}$ from 0.4 to $6.6 \mathrm{dBi}$.

\section{Analysis Of DuAL-CAP Mushroom-LiKe Unit Cell USING EQUIVALENT CIRCUIT}

The proposed dual-cap mushroom-like MS is shown in Fig. 1 which, for illustration purpose, is composed of four unit cells and designed on a doubled-sided substrate. On the top side of the substrate, the unit cells have a square patch with a size of $u 1 \times u 1 \mathrm{~mm}^{2}$ and a shorting via at the center as shown in Fig. 1(a). The unit cell has a size of $(u 1+g l) \times(u 1+g l) \mathrm{mm}^{2}$ and so has a periodicity of $(u 1+g 1)$. On the bottom side, a loop slot having a shape as shown in Fig. 1(b) is etched underneath each patch. It has a cross-section view and an equivalent circuit shown in Figs. 1(c) and (d), respectively, which will be used for analysis later.

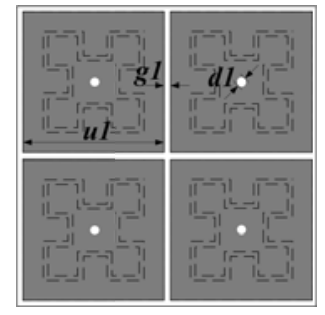

(a)

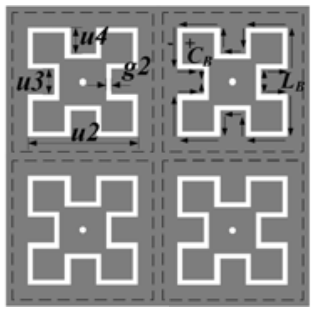

(b)

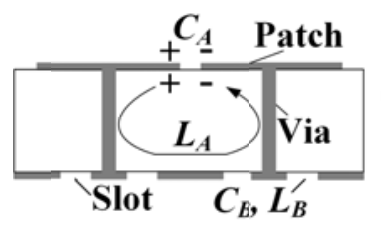

(c)

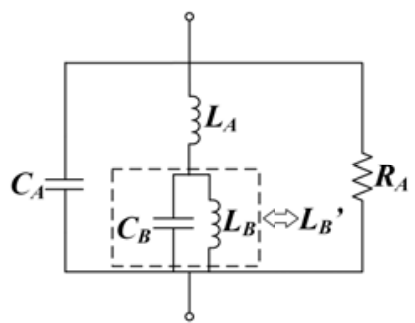

(d)
Fig. 1 Proposed dual-cap mushroom-like unit cells: (a) top view, (b) bottom view, (c) cross-section view, and (d) equivalent circuit. $\left(R_{A}\right.$ represents inherent loss of design)

Conventional unit cells have a square patch with a particular pattern [8-12] on the top side and always have a plain conducting plate on the other side of the substrate. Figs. 2(a) and (b) show the simplest type of conventional mushroom-like MS employing four unit cells having a simple square patch with a centered via on the top side and a plain conducting plate on the bottom side of the substrate. The cross-section view is shown in Fig. 2(c). The fringing coupling between the adjacent patches on the top side generates a capacitance [31]

$$
C_{A}=\frac{u_{1} \varepsilon_{0}\left(1+\varepsilon_{r}\right)}{\pi} \cosh ^{-1}\left(\frac{u_{1}+g_{1}}{g_{1}}\right)
$$

where $\varepsilon_{0}=8.854 \times 10^{-12} \mathrm{farad} / \mathrm{m}$ is the permittivity of freespace, $\varepsilon_{r}=3.66$ is the relative permittivity of the substrate used, and $u_{l}$ and $g_{l}$ are dimensions of the unit cells given in Fig. 1(a). The current loop along the vias and bottom plane generates an inductance [31]

$$
L_{A}=\mu h_{1}
$$

where $\mu$ is about the permeability of free-space, i.e., $\mu=$ $4 \pi \times 10^{-7}$ henry $/ \mathrm{m}$, and $h_{I}=1.6 \mathrm{~mm}$ is the thickness of the substrate. Thus the conventional mushroom-like unit cell can be modeled using a $L C$ resonance circuit as shown in Fig. 2(d) having a resonant frequency [31]

$$
f_{L C}=\frac{1}{2 \pi \sqrt{L_{A} C_{A}}}
$$

The fractional bandwidth of the $L C$ circuit is [32]

$$
B W_{L C}=\sqrt{\frac{L_{A}}{C_{A}}} \frac{1}{R_{A}}
$$

where $R_{A}$ is the inherent power loss of the circuit, as shown in Fig. 2(d).

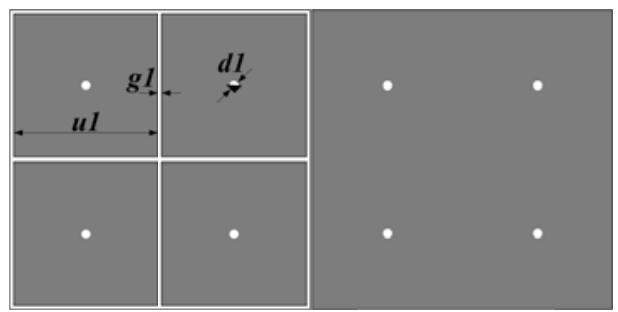

(a)

(b) 


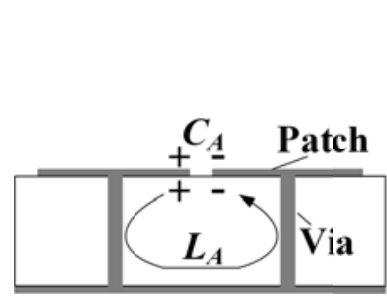

(c)

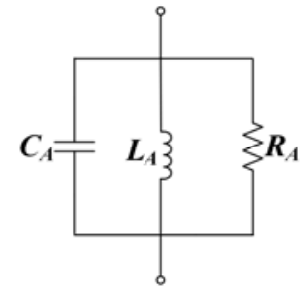

(d)
Fig. 2 Simplest conventional mushroom-like unit cells: (a) top view and (b) bottom view, (c) cross-section view, and (d) equivalent circuit. $\left(R_{A}\right.$ represents inherent loss of design)

For the current dual-cap mushroom-like MS, the crosssection view is shown in Fig. 1(c). The current along the loop slot on the bottom side generates an inductance $L_{B}$ and the fringing coupling between both sides of the loop slot generates a capacitance $C_{B}$. Thus the loop slot on the bottom side is equivalent to another resonant circuit shown in Fig. 1(d) which has a resonant frequency

$$
f_{\text {slot }}=\frac{1}{2 \pi \sqrt{L_{B} C_{B}}}
$$

where the capacitance $C_{B}$ is given by [33]

$$
C_{B}=\frac{1}{2 f_{\text {slot }} Z_{\text {slot }}}
$$

with $Z_{\text {slot }}$ being the characteristic impedance of the loop slot. However, the resonance frequency of the loop slot is also given by [33]

$$
f_{\text {slot }}=\frac{c_{0}}{l_{\text {slot }} \sqrt{\varepsilon_{\text {effs }}}}
$$

where $l_{\text {slot }}$ is the circumference of the loop slot and $\varepsilon_{\text {effs }}=2 \varepsilon_{r} /\left(1+\varepsilon_{r}\right)$ is the effective dielectric constant. Using (5)(7) [33], the inductance $L_{B}$ in the equivalent circuit of Fig. 1(d) can be written as

$$
L_{B}=\frac{1}{\left(2 \pi f_{\text {slot }}\right)^{2} C_{B}}
$$

The impedance of the loop slot on the bottom side is [32]

$$
Z_{\text {slot }}=\frac{j 2 \pi f L_{B}}{1-(2 \pi f)^{2} L_{B} C_{B}}
$$

At frequencies less than or greater than the loop resonant frequency $f_{\text {slot }}$ given by (7), the parallel tuned circuit (having $C_{B}$ and $L_{B}$ ) is inductive or capacitive, respectively. Thus for $f$ $<f_{\text {slot }}$, the impedance $Z_{\text {slot }}$ given by (9) of the parallel tuned circuit is effectively an inductor, which is denoted here as $L_{B}$ ' and shown in Fig. 1(d). Taking into account $L_{A}$ and $C_{A}$, the resonant frequency of the new $L C$ tuned circuit in Fig. 1(d) is

$$
f_{\text {new } L C}=\frac{1}{2 \pi \sqrt{\left(L_{B}^{\prime}+L_{A}\right) C_{A}}}
$$

and the fractional bandwidth is [32]

$$
B W_{\text {new } L C}=\sqrt{\frac{L_{A}+L_{B}^{\prime}}{C_{A}}} \frac{1}{R_{A}}
$$

The resonant frequency $f_{\text {new } L C}$ in (10) determines the operating frequency of the dual-cap mushroom-like unit cells and is lower than $f_{L C}$ given by (3) of conventional mushroom-like unit cells. The fractional bandwidth $B W_{\text {new } L C}$ given by (11) of the dual-cap mushroom-like unit cells is also larger than $B W_{L C}$ given by (4) of conventional mushroom-like unit cells. It should be noted that the condition for having these advantages is that the operating frequency must be less than the slot resonant frequency, i.e., $f<f_{\text {slot }}$. With dimensions $u l=23.6$ $\mathrm{mm}, g l=0.4 \mathrm{~mm}, g 2=0.8 \mathrm{~mm}, u 2=17.2 \mathrm{~mm}, u 3=4.1 \mathrm{~mm}$ and $u 4=4.1 \mathrm{~mm}$ used in (7) for the current unit cells of Fig. 1(b), the slot resonant frequency is computed to be $f_{\text {slot }}=2.346 \mathrm{GHz}$. Using (1), (2), (6) and (8), the circuit parameters $C_{A}, L_{A}, C_{B}$ and $L_{B}$, respectively, are computed and listed in Table I. Using these values in the simulation software ADS, the computed resonant frequency $f_{\text {new } L C}$ for the tuned circuit of Fig. 1(d) is $f_{\text {new } L C}=1.65 \mathrm{GHz}$ which is less than the slot resonant frequency $f_{\text {slot }}=2.346 \mathrm{GHz}$, so the condition $f<f_{\text {slot }}$ is satisfied.

TABLE I

COMPUTEd CiRCUIT PARAMETERS USING (1)-(8) FoR Fig. 1(D)

\begin{tabular}{|c|c|c|c|c|c|c|}
\hline & $C_{A}$ & $L_{A}$ & $C_{B}$ & $L_{B}$ & \multicolumn{2}{|c|}{$f_{0}$ using } \\
\hline Eqn & $(1)$ & $(2)$ & $(5)$ & $(8)$ & ADS & CST \\
\hline Value & $1.48 \mathrm{pF}$ & $2.01 \mathrm{nH}$ & $2.13 \mathrm{pF}$ & $2.16 \mathrm{nH}$ & $1.65 \mathrm{GHz}$ & $1.59 \mathrm{GHz}$ \\
\hline
\end{tabular}

The resonant frequency of the dual-cap mushroom-like unit cells is further studied using CST as follows [2,3]. The MS of Figs. 1(a) and (b) is modeled using the FSS template in CST. An incident wave is applied to a unit cell of the MS. The S parameters of the unit cell is computed using periodic boundary. The resonant frequency is taken as the frequency with a reflection phase of $0^{0}$ and is found to be $1.59 \mathrm{GHz}$ which is also listed in Table I for comparison. Thus the resonant frequency obtained using the equivalent circuit model of Fig. 1(d) differs by only $3.8 \%$, indicating that the equivalent circuit model is quite an accurate model.

Finally, we compare the bandwidth and size of the dual-cap mushroom-like unit cell with those of other unit cells such as the conventional mushroom-like unit cell in [4-7], square patch in [8], UC-PBG lattice in [9], waffle-like unit cell in [10], square loop in [11], and Minkowski fractal mushroomlike unit cell in [12]. For fair comparison, these unit cells are redesigned to operate at the same operating frequency of 1.58 $\mathrm{GHz}$ and on the same type substrate with a relative permittivity of 3.66, a loss tangent of 0.004 and a thickness of $1.6 \mathrm{~mm}$. Their geometries are shown in Fig. 3. Note that they all have square shapes. With the use of the CST, the simulated reflection phases of the unit cells are shown in Fig. 4. The bandwidths, defined as the frequency ranges within $\pm 90^{\circ}$ in the reflection phase curves [8], of these unit cells are shown in Table II. It can be seen that the dual-cap mushroom-like unit cell has a bandwidth of $12.66 \%$ which is much larger than other unit cells, yet having the smallest size of only $576 \mathrm{~mm}^{2}$ $\left(0.19 \lambda_{g}\right)^{2}$. 


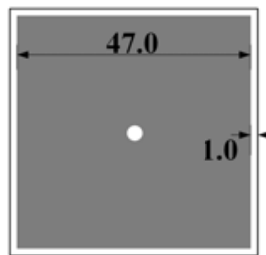

(a)

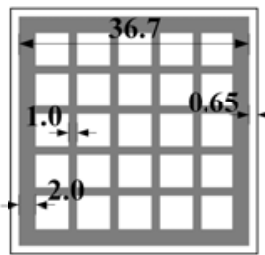

(d)

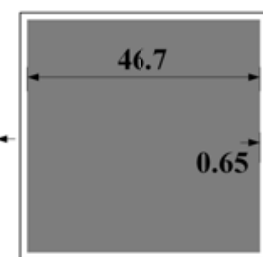

(b)

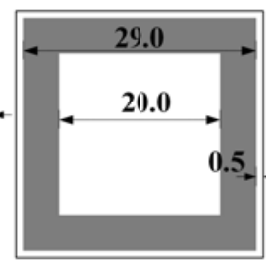

(e)

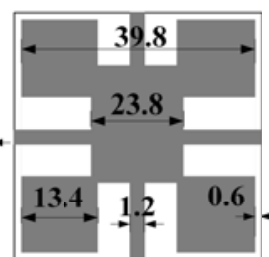

(c)

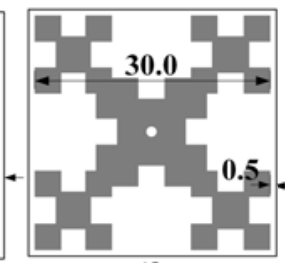

(f)
Fig. 3 Top views of different unit cells: (a) patch with a centered via (conventional mushroom-like unit cell) [4-7], (b) patch with no via [8], (c) UC-PBG lattice [9], (d) waffle-like [10], (e) square loop-like [11], and (f) Minkowski fractal mushroom-like [12] (metal: gray color, substrate: white color) .

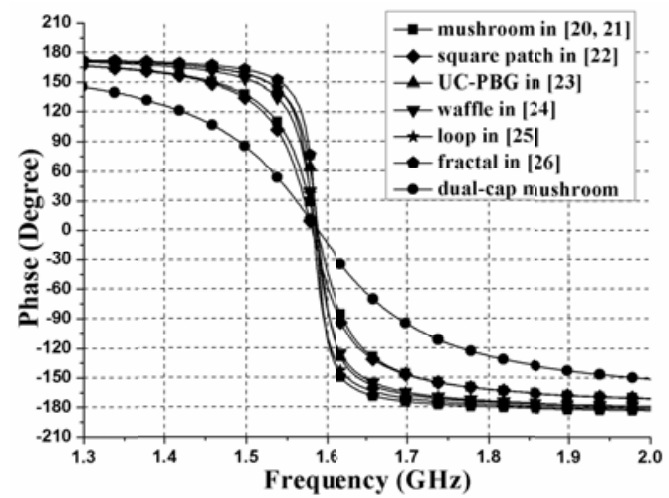

Fig. 4 Reflection phase of different unit cells

TABLE II COMPARISON OF UNIT CELLS AT OPERATING FREQUENCY OF 1.58 $\mathrm{GHz}$

\begin{tabular}{|c|c|c|c|c|c|c|c|}
\hline Unit Cell & $\begin{array}{c}\text { Mushroom } \\
{[4-7]}\end{array}$ & $\begin{array}{c}\text { Patch } \\
{[8]}\end{array}$ & $\begin{array}{c}\text { UC-PBG } \\
{[9]}\end{array}$ & $\begin{array}{c}\text { Waffle } \\
{[10]}\end{array}$ & $\begin{array}{c}\text { Loop } \\
{[11]}\end{array}$ & $\begin{array}{c}\text { Fractal } \\
{[12]}\end{array}$ & $\begin{array}{c}\text { Dual-cap } \\
\text { mushroom }\end{array}$ \\
\hline BW (\%) & 4.43 & 4.37 & 1.90 & 2.28 & 1.65 & 1.27 & 12.66 \\
\hline Size $\left(\mathrm{mm}^{2}\right)$ & 2401 & 2304 & 1681 & 1444 & 900 & 961 & 576 \\
\hline
\end{tabular}

\section{CPR MS ANTENNA}

\section{A. Antenna Structure}

Here the dual-cap mushroom-like MS is used, as shown in Fig. 5(a), to design a CPR planar antenna with high boresight gain and wide operating bandwidth. The MS is placed at the back of the CPR antenna (the source antenna) to serve as a reflector and form a CPR MS antenna. The CPR antenna is similar to our previous design in [29] but with significant reduction in complexity. It is composed of two monopoles, denoted as $M P 1$ and $M P 2$, a switchable feeding network, a Wilkinson-power divider and a defected ground structure (DGS) as shown in Figs. 5(a) and (b). MP1 and $M P 2$ are placed perpendicularly to each other and generating two orthogonal E-field components. The switchable feeding network is composed of a double-pole double-throw (DPDT) switch and a $90^{\circ}$-phase shifter implemented using a $\lambda_{g} / 4-$ microstrip line with the length approximately given by

$$
2 l 4=\lambda_{g} / 4\left(\lambda_{g} \approx c /\left(f_{c} \sqrt{\left(\varepsilon_{r}+1\right) / 2}\right)\right)
$$

The two arms of the Wilkinson-power divider have a characteristic impedance of $1.414 \times Z_{0}$ and a length of

$$
2 l 2+l 3=\lambda_{g} / 4
$$

They serve as $\lambda_{g} / 4$-transformers. A chip resistor with a value of $2 Z_{0}=100 \Omega$ is placed across the outputs of the Wilkinsonpower divider as shown in Fig. 5(a). The power divider splits the input signal from the feed line into two signals with equal amplitude and phase, and feeds them to the phase shifter to produce two signals with a phase difference of $90^{\circ}$. Based on the two DC control voltages (which will be described later), the DPDT switch determines the two signals feeding to $M P 1$ and $M P 2$ will have a phase difference of $90^{\circ}$ or $-90^{\circ}$, hence generating a RHCP or LHCP signal, respectively [34]. The two monopoles are meandered to have a tapered shape for better matching and hence a wider impedance bandwidth. The DGS on the ground plane is an inverted $T$-shaped slot, as shown in Fig. 5(b), serves as a $\lambda_{g} / 4$-slot bandstop filter [35] to reduce coupling between $M P 1$ and $M P 2$ and hence improves their isolation. The horizontal segments of the inverted $T$ shaped slot are meandered to save space. The total length of the slot to both sides is

$$
l 13+2 l 14+2 l 15=\lambda_{g} / 4
$$

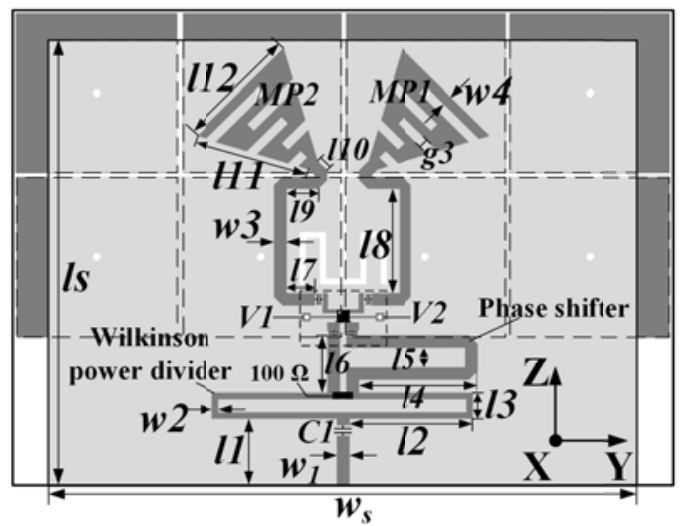

(a)

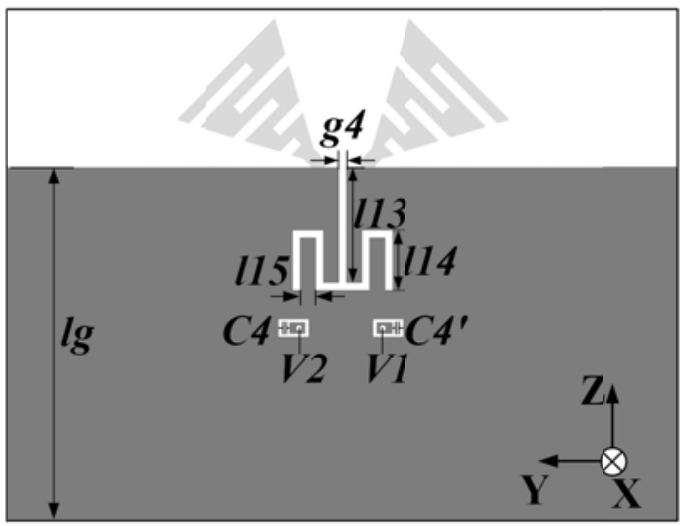

(b) 


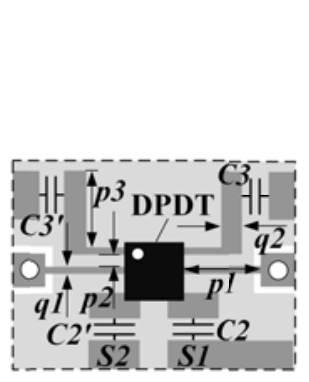

(c)

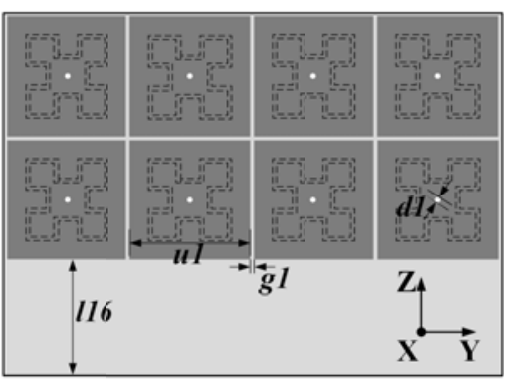

(d)

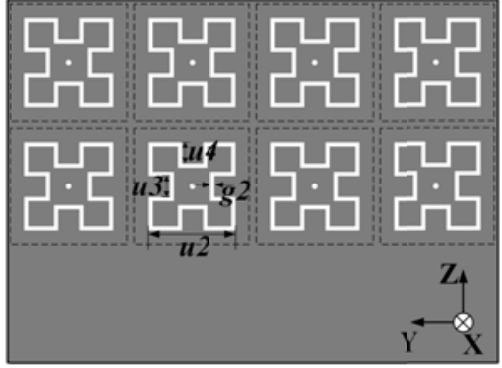

(e)

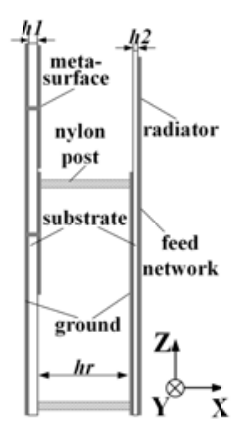

(f)
Fig. 5 (a) Top view of CPR MS antenna, (b) bottom view of CPR antenna, (c) biasing circuit for DPDT switch, (d) top view of MS, (e) bottom view of MS, and (f) side view of CPR MS antenna (metal in front: dark gray color, metal in bottom: light gray color, dashed line: shape in bottom)

The dual-cap mushroom-like MS is designed on a doubledsided substrate and composed of eight square unit cells, as shown in Figs. 5(d) and (e). Each unit cell has a size of $(u l+g l) \times(u l+g l) \mathrm{mm}^{2}$ and so a periodicity of $(u l+g l)$. On the top side of the MS, each unit cell has a patch with a size of $u 1 \times u 1 \mathrm{~mm}^{2}$ and a shorting via at the center. On the bottom side, a loop slot having a shape as shown in Fig. 5(e) is etched underneath each patch. In the CPR MS antenna, the MS is placed at a distance of $h r=0.09 \lambda_{0}$ from the back of the source antenna, as shown in Fig. 5(f), and serving as a reflector to increase the boresight gain and bandwidth of the CPR antenna. The CPR MS antenna has a total volume of $88 \times 69 \times 20.4 \mathrm{~mm}^{3}$.

The EM simulation tool CST is used to study and design the MS and CPR antenna on two substrates with a thickness of 1.6 and $0.8 \mathrm{~mm}$, a relative permittivity of 3.66 and a loss tangent of 0.004 . The final dimensions of the CPR MS antenna are shown in Table III which are used to fabricate the antenna, as shown in Fig. 6. Four nylon posts are used to fix the distance between the source antenna and the MS reflector. The prototyped antenna is measured using the Satimo Starlab system [30].

TABLE III

DIMENSIONS OF PROPOSED CPR ANTENNA (MM)

\begin{tabular}{|c|c|c|c|c|c|c|c|c|c|c|c|c|c|}
\hline$l 1$ & $l 2$ & $l 3$ & $l 4$ & $l 5$ & $l 6$ & $l 7$ & $l 8$ & $l 9$ & $l 10$ & $l 11$ & $l 12$ & $l 13$ & $l 14$ \\
\hline 10.0 & 18.5 & 3.8 & 17.8 & 3.0 & 8.6 & 4.2 & 15.5 & 6.0 & 2.0 & 17.5 & 18.4 & 15.0 & 7.8 \\
\hline$l 15$ & $l 16$ & $l g$ & $g 1$ & $g 2$ & $g 3$ & $g 4$ & $w 1$ & $w 2$ & $w 3$ & $w 4$ & $w s$ & $h 1$ & $h 2$ \\
\hline 2.0 & 22.4 & 46.0 & 0.4 & 0.8 & 1.4 & 1.0 & 1.8 & 0.9 & 1.8 & 1.4 & 88.0 & 1.6 & 0.8 \\
\hline$h r$ & $l s$ & $p 1$ & $p 2$ & $p 3$ & $q 1$ & $q 2$ & $u 1$ & $u 2$ & $u 3$ & $u 4$ & $d 1$ & & \\
\hline 18.0 & 69.0 & 4.3 & 0.25 & 2.6 & 0.25 & 0.8 & 23.6 & 17.2 & 4.1 & 4.1 & 0.6 & & \\
\hline
\end{tabular}

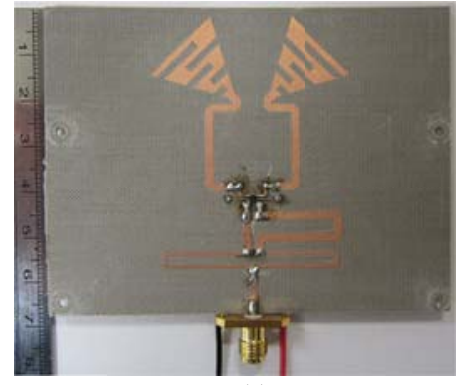

(a)

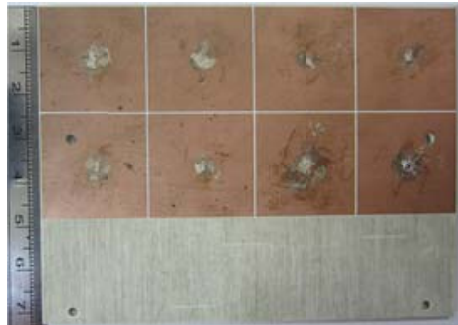

(c)

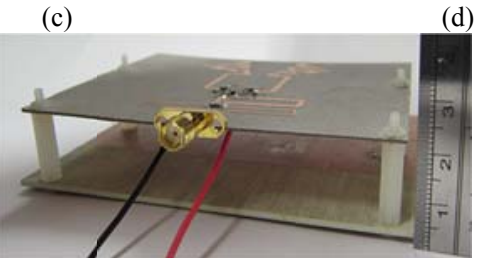

(e)

Fig. 6 Prototyped CPR MS antenna: (a) top view of CPR antenna, (b) bottom view of CPR antenna, (c) top view of MS, (d) bottom view of MS, and (e) side view of CPR MS antenna

\section{B. Biasing Circuit}

A practical DPDT switch, SKY13355-374LF, from Skyworks is used in our design [36]. The switch has a Micro Leadframe Package Dual (MLPD) with six pins and a physical dimension of $1.5 \times 1.5 \times 0.5 \mathrm{~mm}^{3}$. For clarity, the enlarged version of the biasing circuit for the DPDT switch in Fig. 5(a) shown in Fig. 5(c), where six microstrip lines are designed to connect to the six pins of the DPDT. The input signals $S 1$ and $S 2$ are obtained from the phase shifter with $S 1$ being lagging by $90^{\circ}$, as can be seen in Fig. 5(a). The capacitors, $C 2, C 2$ ', $C 3$ and $C 3$ ' with a value of $100 \mathrm{pF}$, placed between each pin of DPDT switch and the corresponding microstrip line, are used for DC blocking. The state of the DPDT switch is determined by the control DC voltages $V 1$ and $V 2$ at pins 2 and 5 , respectively, as shown in Fig. 7. With $V I=3 \mathrm{~V}$ and $V 2=0 \mathrm{~V}$, input signals $S 1$ and $S 2$ are fed to $M P 1$ and $M P 2$, respectively, as shown in Fig. 7(a), so the signal to $M P 1$ is lagging the to $M P 2$ by $90^{\circ}$, resulting in RHCP. While with $V 1=0 \mathrm{~V}$ and $V 2=3 \mathrm{~V}$, signals $S 1$ and $S 2$ will be fed to $M P 2$ and $M P 1$, respectively, as shown in Fig. 7(b), and the signal to $M P 2$ will be lagging the signal to $M P 1$ by $90^{\circ}$, resulting in LHCP. In 5 (c), the DC voltage $V 2$ to pin 5 of the DPDT switch is via a microstrip strip $p 1$ which has a DC pad at the other end. Another DC pad is printed in exactly the same position on the ground plane as shown in Fig. 5(b) where capacitor $C 4$ with a value of $10 \mathrm{pF}$ is used for DC decoupling. These two DC pads are connected together through a via. To control the DPDT switch, two DC voltage $V 1$ and $V 2$ are applied to the pads on ground plane using two wires, as shown in Fig. 6(b). Thus the 
DC wires are placed on the ground plane, minimizing the effects on the antenna performance. The way that the DC voltage $V 1$ supplied to pin 2 of the DPDT switch is exactly the same as that for $V 2$ to pin 5 , so the description is not repeated here. Table IV shows the DC control voltages of the DPDT switch for the two CPs of the antenna. Note that the antenna requires only two DC control voltages. Compared with the previous design in [29], the design complexity and cost are substantially reduced.

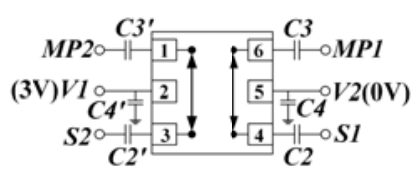

(a)

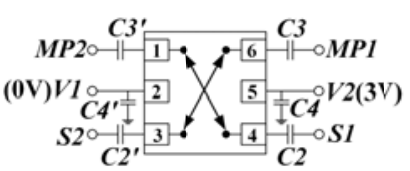

(b)
Fig. 7 Operating diagram of DPDT switch for antenna in (a) RHCP and (b) LHCP

TABLE IV DC CONTROL VOLTAGES FOR TWO POLARIZATIONS

\begin{tabular}{|c|c|c|c|c|c|c|}
\hline & $V 1$ & $V 2$ & $\begin{array}{l}\text { MP1 (Pin 6) } \\
\text { to S1 (Pin 4) }\end{array}$ & $\begin{array}{l}\text { MP1 (Pin 6) } \\
\text { to } 2 \text { (Pin 3) }\end{array}$ & $\begin{array}{l}\text { MP2 (Pin 1) } \\
\text { to S1 (Pin 4) }\end{array}$ & $\begin{array}{l}\text { MP2 (Pin 1) } \\
\text { to S2 (Pin 3) }\end{array}$ \\
\hline RHCP & $3 \mathrm{~V}$ & $0 \mathrm{~V}$ & ON & OFF & OFF & ON \\
\hline LHCP & $0 \mathrm{~V}$ & $3 \mathrm{~V}$ & OFF & ON & ON & OFF \\
\hline
\end{tabular}

\section{EFFECTS OF MS ON CPR ANTENNA}

In the setup of the CPR MS antenna shown in Fig. 5(f), the distance between the MS and the source antenna is optimized, in terms of operating bandwidth, to be $h r=0.09 \lambda_{0}=18 \mathrm{~mm}$ using the CST. The simulated S11, AR and realized boresight gain of the CPR MS antenna are shown in Fig. 8, indicating that the antenna has an impedance bandwidth (IMBW) for $\mathrm{S} 11<-10 \mathrm{~dB}$ of 1.21-2.09 GHz, axial-ratio bandwidth (ARBW) for $\mathrm{AR}<3 \mathrm{~dB}$ of $1.42-1.88 \mathrm{GHz}$, and a boresight gain of 6.6 $\mathrm{dBi}$. For comparison, the simulated results using a simple conducting plate, i.e., a single-side $\mathrm{PCB}$, as the reflector are also shown in the figure. It can be seen that both types of reflectors result in the similar IMBWs and ARBWs, but the one using the simple conducting plate has a lower boresight gain of $3.8 \mathrm{dBi}$. The results without using any reflector are also shown in the same figure for comparison. In such case, the CPR antenna needs to be re-optimized again in terms of operating bandwidth. It can be seen that, without using any reflector, the IMBW and boresight gain reduce to 1.30-1.79 $\mathrm{GHz}$ and $0.4 \mathrm{dBi}$, respectively, with the ARBW remaining about same.

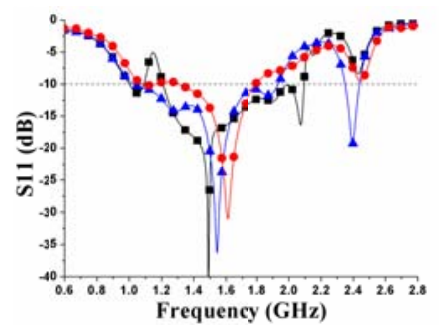

(a)

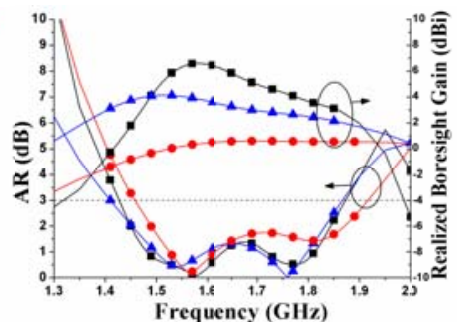

(b)
Fig. 8 Simulated (a) S11, and (b) AR and realized boresight gain with MS reflector, with simple conducting reflector and without reflector $(-\longrightarrow$ : MS reflector; $-\mathbf{-}-$ : simple conducting reflector; $-\boldsymbol{-}-$ : without reflector)

The operating bandwidth of a $\mathrm{CP}$ antenna is the overlapping bandwidth of the IMBW and ARBW, and is an important property. The source antenna in the current CPR MS antenna similar to the one in [29] which had presented the parametric study to set the IMBW and ARBW, so the parametric study is not repeated here. Instead, we present the results of the parameter study on the MS reflector.

In the geometry of the MS shown in Figs. 5(d) and (e), there are many parameters such as $u 1-u 4, g 1, g 2, d 1, h r$ which can affect the performance of the CPR MS antenna. However, simulation has shown that lengths $u 1$ and $u 2$ of the patch and slot pattern, respectively, of the unit cell and the distance $h r$ between the source antenna and the MS have significant effects on the IMBW and ARBW. Thus the results of parametric study on these parameters in RHCP (which are the same in LHCP) are presented.

The simulated S11 with different values of $u 1, u 2$, and $h r$ are shown in Figs. 9(a), (b), and (c), respectively. With $u 1$ increased from $22.6 \mathrm{~mm}$ to 23.6 and $24.6 \mathrm{~mm}$, Fig. 9(a) shows that the low-cutoff frequency of the IMBW remains almost unchanged, and the high-cutoff frequency increases from 1.96 $\mathrm{GHz}$ to 1.99 and $2.07 \mathrm{GHz}$. With $u 2$ increased from $16.2 \mathrm{~mm}$ to 17.2 and $18.2 \mathrm{~mm}$, Fig. 9(b) shows that the low-cutoff frequency of the IMBW remains almost unchanged, and the high-cutoff frequency increases from $1.97 \mathrm{GHz}$ to 1.99 and $2.07 \mathrm{GHz}$. With $h r$ reduced from $24 \mathrm{~mm}$ to 18 and $12 \mathrm{~mm}$, Fig. 9(c) shows that the high-cutoff frequency slightly decreases from $2.06 \mathrm{GHz}$ to 1.99 and $1.96 \mathrm{GHz}$. However, when it is further reduced to $12 \mathrm{~mm}$, mismatching is observed. Thus $u 1$ and $u 2$ can be used to set the high-cutoff frequency.

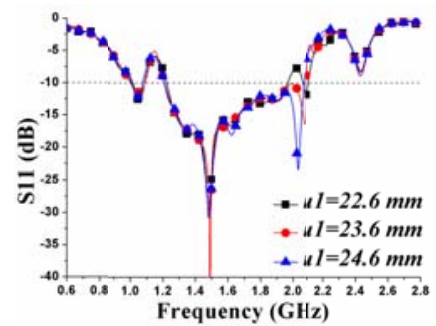

(a)

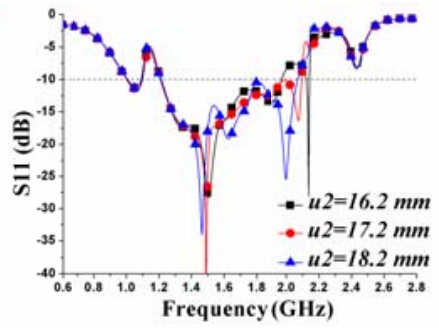

(b)

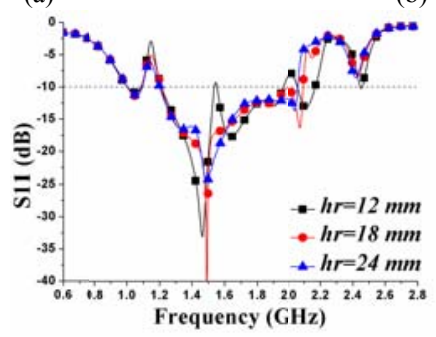

(c)

Fig. 9 Simulated S11 with different (a) $u 1$, (b) $u 2$, and (c) $h r$ in RHCP

The simulated ARs with different values of $u 1, u 2$ and $h r$ shown in Figs. 10(a), (b) and (c), respectively. With $u 1$ increased from $22.6 \mathrm{~mm}$ to 23.6 and $24.6 \mathrm{~mm}$, the ARBW remains almost unchanged, as shown in Fig. 10(a). With $u 2$ increased from $16.2 \mathrm{~mm}$ to 17.2 and $18.2 \mathrm{~mm}$, the ARBW from $1.44-1.89$ to $1.42-1.88$ and $1.40-1.83 \mathrm{GHz}$, as shown in Fig. 10(b). With $h r$ decreased from $24 \mathrm{~mm}$ to 18 and $12 \mathrm{~mm}$, Fig. 10(c) shows that the ARBW and the center frequency 
remain about the same. But when the $\mathrm{MS}$ is at a closer of $h r=12 \mathrm{~mm}$ from the source antenna, the AR within the ARBW rises. These results show that $u 2$ has significant effects on the magnitude of AR and ARBW, $h r$ affects the AR within the ARBW, and $u 1$ has little effects on the ARBW.

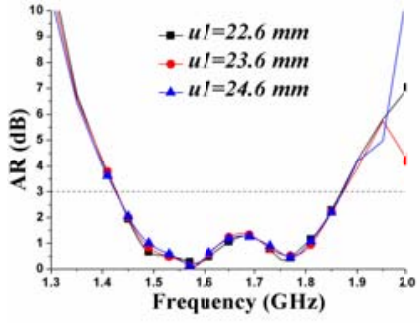

(a)

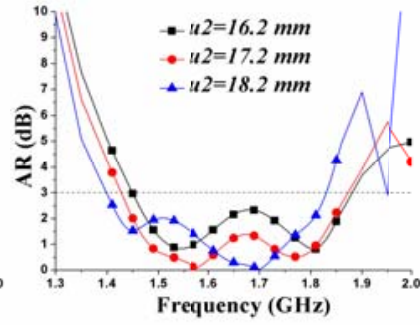

(b)

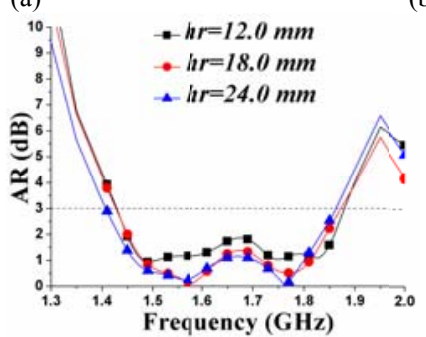

(c)

Fig. 10 Simulated AR with different (a) $u 1$, (b) $u 2$, and (c) $h r$ in RHCP

It is important to be able to use the design in other frequency bands. Based on the above results, we propose the design methodology of the proposed CPR MS antenna for other frequency bands as follows:

1. Follow the design procedure in [29] to design the CPR antenna for the desirable operating frequency.

2. Select $u 1$ and $u 2$ using (1)-(8) to design the dual-cap mushroom-like MS for the desirable operating frequency.

3. Place the MS at a distance of about $\lambda_{0} / 4$ away from the back of the source antenna, i.e, the CPR antenna.

4. Adjust $u 2$ to obtain the required AR (see Fig. 10(b)).

5. Adjust $u l$ to obtain the required IMBW (see Fig. 9(a)).

6. Adjust $h r$ to obtain better AR and matching.

Here, it should be pointed out that the dual-cap mushroomlike can be used with any other planar antennas and not only this CPR antennas.

\section{Simulation And Measurement Results}

\section{A. S11 and AR}

The simulated and measured S11 and AR of the CPR MS antenna in RHCP and LHCP are shown in Fig. 11. It can be seen in Fig. 11(a) that, in both RHCP and LHCP, the simulated IMBWs are 1.21-2.09 GHz $(0.88 \mathrm{GHz}, 53.3 \%)$. The measured IMBWs in RHCP and LHCP are 1.22-2.19 GHz $(0.97 \mathrm{GHz}, 56.7 \%)$ and $0.97-2.19 \mathrm{GHz}(1.22 \mathrm{GHz}, 77.2 \%)$, respectively. Fig. 11(b) shows that the simulated ARBWs in both polarizations for $\mathrm{AR}<3 \mathrm{~dB}$ are $1.42-1.88 \mathrm{GHz}(0.46 \mathrm{GHz}$, $27.9 \%$ ). While the measured ARBWs in RHCP and LHCP are 1.43-1.88 GHz $(0.45 \mathrm{GHz}, 27.2 \%)$ and 1.42-1.92 GHz $(0.50$ $\mathrm{GHz}, 29.9 \%$ ), respectively, which are smaller than the IMBW. Thus the measured operating bands of the CPR MS antenna in RHCP and LHCP are 1.43-1.88 GHz and $1.42-1.92 \mathrm{GHz}$, respectively, which are large enough for the GNSS system.

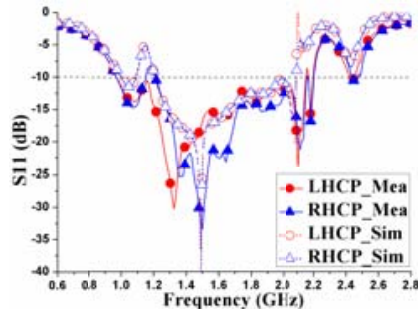

(a)

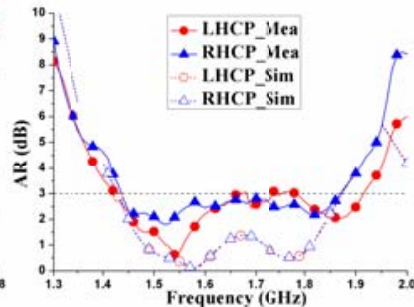

(b)
Fig. 11 Simulated and measured (a) S11 and (b) AR with antenna in RHCP and LHCP

The AR as a function of the elevation $(\theta)$ and azimuth $(\phi)$ angles has also been studied because it is essential for CP antennas. Note that in both RHCP and LHCP, the boresight direction is at $\theta=+90^{\circ}$ and $\phi=0^{\circ}$ and the opposite boresight direction is at $\theta=-90^{\circ}$ and $\phi= \pm 180^{\circ}$. At $1.58 \mathrm{GHz}$ (the center frequency for GNSS), the simulated and measured ARs of the CPR MS antenna with different $\theta$ in the $x-z$ plane and different $\phi$ in the $x-y$ plane are shown in Fig. 12. In the $x-z$ plane, Fig. 12(a) shows that the simulated AR beamwidth (for $\mathrm{AR}<3 \mathrm{~dB})$ is $48^{0}<\theta<141^{\circ}\left(93^{\circ}\right)$ in both RHCP and LHCP. The measured beamwidths are $66^{\circ}<\theta<94^{0}\left(28^{0}\right)$ and $52^{0}<\theta<100^{\circ}\left(48^{0}\right)$ in RHCP and LHCP, respectively. In the $\mathrm{x}-$ y plane, the simulated AR beamwidths are $-41^{0}<\phi<32^{0}\left(73^{0}\right)$ and $-32^{\circ}<\phi<41^{0}\left(73^{\circ}\right)$ in RHCP and LHCP, respectively, and the measured AR beamwidths are from $-34^{0}<\phi<12^{0}\left(46^{\circ}\right)$ and from $-34^{0}<\phi<30^{\circ}\left(64^{\circ}\right)$ in RHCP and LHCP, respectively. The differences between the simulation and measurement are mainly due to the fabrication, prototyping and measurement tolerances. Thus the simulated beamwidths of the antenna are about $93^{\circ}$ in the $\mathrm{x}-\mathrm{z}$ plane and $73^{\circ}$ in the $\mathrm{x}-\mathrm{y}$ plane.

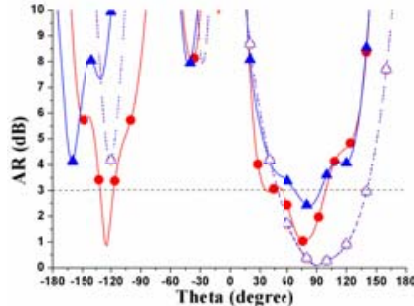

(a)

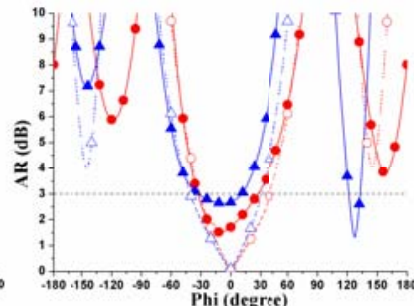

(b)
Fig. 12 Simulated and measured AR in (a) x-z plane and (b) $x-y$ plane at 1.58 $\mathrm{GHz}$ (measured results with antenna in RHCP: -1 and LHCP: simulated results with antenna in RHCP: $-\triangle$ - and LHCP: - $\bigcirc_{-}$)

\section{B. Radiation Patterns}

The measured and simulated radiation patterns of the CPR MS antenna at $1.58 \mathrm{GHz}$ in RHCP and LHCP are shown in Fig. 13. Good agreements can be observed. When the antenna is in RHCP, co-polarization is clockwise circular and crosspolarization is anti-clock circular, and vice versa for the antenna in LHCP. With the antenna in RHCP, Fig. 13(a) shows that the radiation patterns of co-polarization point at the boresight direction. The radiation patterns of crosspolarization have a much lower amplitude at the opposite direction. With the antenna in LHCP, Fig. 13(b) shows that the radiation patterns are in opposite directions to those in Fig. 13(a). 


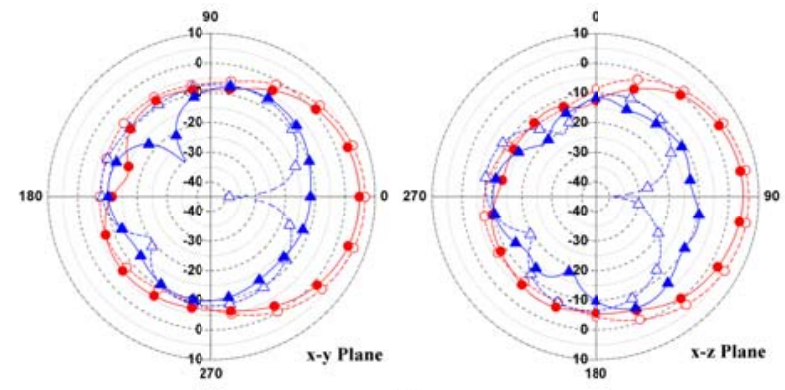

(a)

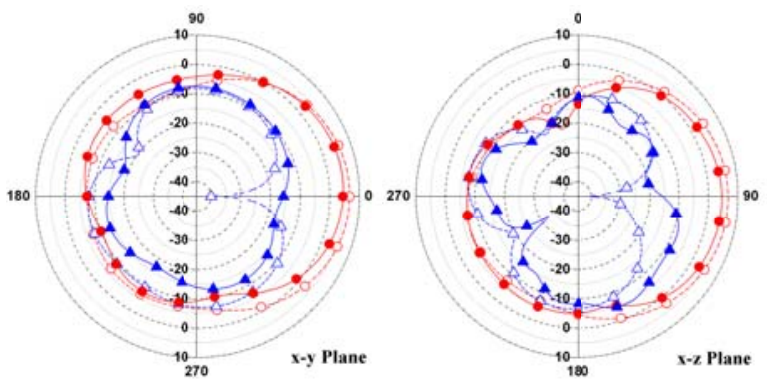

(b)

Fig. 13 Simulated and measured radiation patterns at $1.58 \mathrm{GHz}$ with antenna (a) RHCP and (b) LHCP (simulated co-polarization:- $\mathrm{O}^{-}$, and crosscross-polarization: $-\triangle-;$ measured co-polarization:- - and crosscross-polarization: $-\boldsymbol{\sim}$ )

\section{Realized Boresight Gains}

The simulated and measured realized boresight gains of the CPR MS antenna are shown in Fig. 14. In RHCP and LHCP, the simulated boresight gain in co-polarization ranges from 0.2 to $6.6 \mathrm{dBi}$ across the operating band of $1.42-1.88 \mathrm{GHz}$. In $\mathrm{RHCP}$, the measured boresight gain in co-polarization ranges from -1.2 to $5.3 \mathrm{dBi}$ across the operating band of $1.43-1.88$ $\mathrm{GHz}$. In LHCP, the measured boresight gain in co-polarization ranges from -2.3 to $5.0 \mathrm{dBi}$ across the operating band of 1.42$1.92 \mathrm{GHz}$. The differences between the simulated and measured results are mainly due to the fabrication, prototyping and measurement tolerances, and also the cable effects [37]. The simulated and measured boresight gains in crosspolarization are lower than $-10 \mathrm{dBi}$ in both RHCP and LHCP, as shown in Fig. 14.

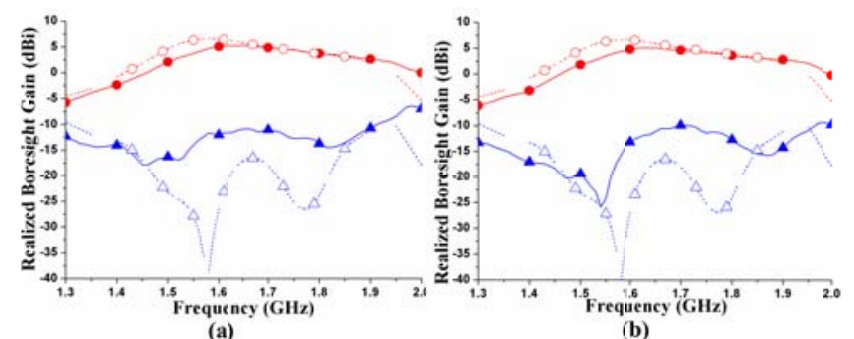

Fig. 14 Simulated and measured realized boresight gains when antenna in (a) RHCP and (b) LHCP (simulated co-polarization:- - , simulated crosscross-polarization: $-\triangle-$, measured co-polarization: $-\bullet$, measured crosscross-polarization: $-\mathbf{-}$ )

\section{Comparison with CPR in [29]}

Finally, the operating frequency bandwidth, realized boresight gain (at $1.58 \mathrm{GHz}$ ), and complexity of biasing circuit of the CPR MS antenna are compared with those of our previous design [29] and results are shown in Table V. It can seen that by using the dual-cap mushroom-like MS, the CPR
MS antenna has a much higher boresight gain of $6.6 \mathrm{dBi}$ and operating bandwidth of $27.9 \%$. With the use of the DPDT switch, the DC biasing circuit requires only 2 DC wires and 9 lumped components (including the capacitors, resistor, and the DPDT), instead of 5 DC wires and 18 lumped components required in the previous design in [29]. Thus the complexity is much reduced.

TABLE V COMPARISON WITH PREVIOUS WORK

\begin{tabular}{|c|c|c|c|c|c|}
\hline Ref & $\begin{array}{c}\text { Center } \\
\text { Frequency } \\
(\mathrm{GHz})\end{array}$ & $\begin{array}{c}\text { Ope BW } \\
(\%)\end{array}$ & $\begin{array}{c}\text { Realized Boresight } \\
\text { Gain }(\mathrm{dBi})\end{array}$ & $\begin{array}{c}\text { No. of DC } \\
\text { Feed Lines }\end{array}$ & $\begin{array}{c}\text { No. of } \\
\text { lumped } \\
\text { Comannanane }\end{array}$ \\
\hline$[29]$ & 1.55 & 15.4 & $-0.2(1.55 \mathrm{GHz})$ & 5 & 18 \\
\hline Pro & 1.65 & 27.9 & $6.6(1.58 \mathrm{GHz})$ & 2 & 9 \\
\hline
\end{tabular}

\section{CONCLUSION}

A new type of MS, known as dual-cap mushroom-like MS, has been proposed and used in the design of a CPR MS antenna. The unit cells on the MS have a simple patch on one side of the substrate and a meander slot on the other side. Equivalent circuit has been derived to analyze the unit cell and verified using the software ADS and CST. The operating bandwidth and size of the dual-cap mushroom-like unit cell have been compared with other unit cells found in literature and results have shown that the dual-cap mushroom-like unit cell has the widest operating bandwidth and smallest size. The dual-cap mushroom-like MS has been used as a reflector in the design of a CPR MS antenna for the GNSS system. Results have shown that the dual-cap mushroom-like MS placed at a distance of $0.09 \lambda_{0}$ away can substantially increase the operating bandwidth and boresight gain of the CPR antenna.

\section{REFERENCES}

[1] C. L. Holloway, E. F. Kuester, J. A. Gordon, J. O. Hara, J. Booth, and D. R. Smith, "An overview of the theory and applications of metasurfaces: the two-dimensional equivalents of metamaterials," IEEE Antennas Propag. Mag., vol. 54, no. 2, pp. 10-35, 2012.

[2] H. L. Zhu, K. L. Chung, X. L. Sun, S. W. Cheung, and T. I. Yuk, "CP metasurfaced antennas excited by LP sources," in IEEE Antennas and Propagation Society International Symposium (APSURSI), pp. 1-2, 2012, Chicago, Illinois, U.S.A.

[3] H. L. Zhu, S. W. Cheung, K. L. Chung, and T. I. Yuk, "Linear-tocircular polarization conversion using metasurface," IEEE Trans. Antennas Propag., vol. 61, no. 9, pp. 4615-4623, Sep. 2013.

[4] F. Yang and Y. Rahmat-Samii, "A low-profile circularly polarized curl antenna over an electromagnetic bandgap EBG surface," Microw. Opt. Tech. Lett., vol. 31, no. 4, pp. 264-267, Nov. 2001.

[5] W. Liu, Z. N. Chen, and X. Qing, "Metamaterial-based low-profile broadband mushroom antenna," IEEE Trans. Antennas Propag., vol. 62, no. 3, pp. 1165-1172, Mar. 2014.

[6] D. Qu, L. Shafai and A. Foroozesh, "Improving microstrip patch antenna performance using EBG substrates," IEE Proc.-Microw. Antennas Propag., vol. 153, no. 6, pp. 558-563, Dec. 2006.

[7] Y. Rahmat-Samii and H. Mosallaei, "Electromagnetic band-gap structures: classification, characterization, and applications," 11st International Conference on Antennas and Propagation, vol.2, pp. 560564, 2001.

[8] A. P. Feresidis, G. Goussetis, S. H. Wang, and J. C. Vardaxoglou, "Artificial magnetic conductor surfaces and their application to lowprofile high-gain planar antennas," IEEE Trans. Antennas Propag., vol. 53, no. 1, pp. 209-215, Jan. 2005.

[9] R. Coccioli, F. R. Yang, K. P. Ma, and T. Itoh, "Aperture-coupled patch antenna on UC-PBG substrate," IEEE Trans. Microw. Theory Tech., vol. 47, no. 11, pp. 2123-2130, Nov. 1999. 
[10] B. S. Cook, and A. Shamim, "Utilizing wideband AMC structures for high-gain inkjet-printed antennas on lossy paper substrate," IEEE Antennas Wireless Propag. Lett., vol. 12, pp. 76-79, 2013.

[11] N. Mohamed Mohamed-Hicho, E. Antonino-Daviu, M. Cabedo-Fabrés, and M. Ferrando-Bataller, "Design of a compact low profile high-gain UHF antenna using high-impedance surfaces," IEEE Antennas and Propagation Society International Symposium (APSURSI), pp. 17741775, 2014, Memphis, Tennessee, USA.

[12] X. L. Bao, G. Ruvio, M. J. Ammann, and M. John, "A novel GPS patch antenna on a fractal hi-impedance surface substrate," IEEE Antennas Wireless Propag. Lett., vol. 5, pp. 323-326, 2006.

[13] S. Gao, A. Sambell, and S. S. Zhong, "Polarization-agile antennas," IEEE Antennas Propag. Mag., vol. 48, no. 3, pp. 28-37, Jun. 2006.

[14] A. Khaleghi and M. Kamyab, "Reconfigurable single port antenna with circular polarization diversity," IEEE Trans. Antennas Propag., vol. 57, no. 2, pp. 555-559, Feb. 2009.

[15] Y. Sung, "Investigation into the polarization of asymmetrical feed triangular microstrip antennas and its application to reconfigurable antennas," IEEE Trans. Antennas Propag., vol. 58, no. 4, pp. 10391046, Apr. 2010.

[16] A. Khidre, K. F. Lee, F. Yang, and A. Z. Elsherbeni, "Circular polarization reconfigurable wideband E-shaped patch antenna for wireless applications," IEEE Trans. Antennas Propag., vol. 61, no. 2, pp. 960-964, Feb. 2013.

[17] S. Pyo, J. W. Baik and Y. S. Kim, "Slot-perturbed microstrip antenna for switchable circular polarization," Electron Lett., vol. 47 no. 10, pp. 583585, May. 2011.

[18] M. S. Nishamol, V. P. Sarin, D. Tony, C. K. Aanandan, P. Mohanan, and K. Vasudevan, "An electronically reconfigurable microstrip antenna with switchable slots for polarization diversity," IEEE Trans. Antennas Propag., vol. 59, no. 9, pp. 3424-3427, Sep. 2011.

[19] X. X. Yang, B. C. Shao, F. Yang, A. Z. Elsherbeni, and B. Gong, "A polarization reconfigurable patch antenna with loop slots on the ground plane," IEEE Antennas Wireless Propag. Lett., vol. 11, pp. 69-72, 2012.

[20] Z. X. Yang, H. C. Yang, J. S. Hong, and Y. Li, "Bandwidth enhancement of a polarization reconfigurable patch antenna with stairslots on the ground," IEEE Antennas Wireless Propag. Lett., vol. 13, pp. 579-582, 2014.

[21] J. F. Tsai and J. S. Row, "Reconfigurable square-ring microstrip antenna," IEEE Trans. Antennas Propag., vol. 61, no. 5, pp. 2857-2860, May 2013.

[22] J. S. Row and M. J. Hou, "Design of polarization diversity patch antenna based on a compact reconfigurable feeding network," IEEE Trans. Antennas Propag., vol. 62, no. 10, pp. 5349- 5352, Oct. 2014.
[23] M. K. Fries, M. Gräni, and R. Vahldieck, "A reconfigurable slot antenna with switchable polarization," IEEE Microw. Wireless Comp. Lett., vol. 13, no. 11, pp. 490-492, Nov. 2003.

[24] W. M. Dorsey, A. I. Zaghloul, and M. G. Parent, "Perturbed square-ring slot antenna with reconfigurable polarization," IEEE Antennas Wireless Propag. Lett., vol. 8, pp. 603-606, 2009.

[25] J. S. Row and C. J. Shih, "Polarization-diversity ring slot antenna with frequency agility," IEEE Trans. Antennas Propag., vol. 60, no. 8, pp. 3953-3957, Aug. 2012.

[26] J. S. Row, W. L. Liu, and T. R. Chen, "Circular polarization and polarization reconfigurable designs for annular slot antennas," IEEE Trans. Antennas Propag., vol. 60, no. 12, pp. 5998-6002, Dec. 2012.

[27] Y. B. Chen, Y. C. Jiao, and F. S. Zhang, "Polarization reconfigurable CPW-fed square slot antenna using pin diodes," Microw. Opt. Tech. Lett., vol. 49, no. 6, pp.1233-1236, Jun. 2007.

[28] H. L. Zhu, S. W. Cheung, X. H. Liu, and T. I. Yuk, "Design of polarization reconfigurable antenna using metasurface," IEEE Trans. Antennas Propag., vol. 62, no. 6, pp. 2891-2898, Jun. 2014.

[29] Y. F. Cao, S. W. Cheung, and T. I. Yuk, "A simple planar polarization reconfigurable monopole antenna for GNSS/PCS," IEEE Trans. Antennas Propag., vol. 63, no. 2, pp. 500-507, Feb. 2015.

[30] http://www.satimo.com/

[31] F. Yang and Y. Rahmat-Samii, "Electromagnetic band gap structures in antenna engineering," Cambridge University Press, 2009, ch. 3, pp. 5963.

[32] D. M. Pozar, "Microwave engineering," John Wiley \& Sons, (4th Edition), 2012, ch. 6, pp. 272-278.

[33] L. H. Hsieh, and K. Chang, "Equivalent lumped elements $G, L, C$, and unloaded $Q$ 's of closed- and open-loop ring resonators," IEEE Trans. Microw. Theory Tech., vol. 50, no. 2, pp. 453-460, Feb. 2002.

[34] C. A. Balanis, "Antenna theory - analysis and design," John Wiley \& Sons, (3rd Edition), 2005, ch. 2, pp. 73-74.

[35] S. Zhang, S. N. Khan and S. He, "Reducing mutual coupling for an extremely closely-packed tunable dual-element PIFA array through a resonant slot antenna formed in-between," IEEE Trans. Antennas Propag., vol. 58, no. 8, pp. 2771-2776, Aug. 2010.

[36] Datasheet for SKY13355-374LF: 0.1-6.0 GHz DPDT switch, Skyworks Solutions, Inc., Oct. 11, 2011.

[37] L. Liu, Y.F. Weng, S. W. Cheung, T. I. Yuk and L. J. Foged, "Modeling of cable for measurements of small monopole antennas," Loughborough Antennas \& Propagation Conference, 14-15 Nov. 2011, Loughborough, (LAPC 2011), UK. 